\title{
Do Stents Interfere With the Densitometric Assessment of a Coronary Artery Lesion?
}

\author{
B.H. Strauss, MD, B.J. Rensing, MD, A. den Boer, MD, W.J. van der Giessen, MD, \\ J.H.C. Reiber, MD, and P.W. Serruys, MD
}

\begin{abstract}
This in vitro study was designed to assess the contribution of three currently investigated coronary stents to the densitometric measurement of a known stenosis contained within two different sized plexiglass phantoms. These studies were performed at two concentrations of the contrast agent iopamidol (50 and 100\%). The calculated minimal luminal cross-sectional area values in the control phanton ranged from $0-18 \%$ higher than the theoretical values. Insertion of a stainless steel stent (Wallstent ${ }^{\circledR}$, Schneider, Zürich or Palmaz-Schatz ${ }^{(m)}$, Johnson and Johnson, Warren, NJ) resulted in further minor increases $(\leq 8 \%$ in the calculated minimal luminal cross-sectional area, except in the smaller phantom filled with $50 \%$ contrast medium. The Wiktor ${ }^{(\mathbb{M})}$ (tantalum) stent (Medtronic, Minneapolis, MN) had the largest impact of the three stents depending on the concentration of iopamidol ( $100 \%$ contrast medium: $9-13 \%$ values above control; $50 \%$ contrast medium; $23-56 \%$ higher). We conclude that although densitometry may overestimate the minimal luminal cross-sectional area in stented vessels, this effect is usually minor with stainless steel stents. However, tantalum-containing stents may result in serious overestimation of lesion area, particularly if contrast is diluted or the vessel is not well filled.
\end{abstract}

Key words: coronary stents, densitometry, tantalum, stainless steel, quantitative coronary angiography

\section{INTRODUCTION}

Coronary stenting has been introduced as an adjunct or alternative to percutaneous transluminal coronary angioplasty (PTCA) in obstructive coronary artery disease $[1,2]$. A variety of devices have been developed which differ in their composition, design, radiopacity, and mode of expansion. Although densitometry and contour detection appear to be comparable methods to assess the angiographic result after stenting with the Wallstent ${ }^{\circledR}[3]$, the actual contribution of the individual types of stent to the densitometric value has not been systematically studied. The objective of this study was to assess whether the physical properties of three devices currently under investigation affect the densitometric assessment of a fixed stenosis in a plexiglass phantom.

\section{MATERIALS AND METHODS}

\section{Plexiglass Phantoms}

Plexiglass models were $6 \mathrm{~cm}$ in length and circular in cross-section. The extremities of the phantoms had a fixed bore diameter of either 4 or $3 \mathrm{~mm}$ and abruptly tapered in the central part to a fixed concentric stenosis of 3 and $2 \mathrm{~mm}$, respectively.

\section{Stent Types}

The Wallstent ${ }^{\circledR}$ (Schneider, Zürich) is a self-expandable stainless steel woven mesh prosthesis, constructed of 16 wire filaments, each $0.07 \mathrm{~mm}$ wide. It is constrained in an elongated configuration on delivery catheter with the distal end covered by a removable plastic sleeve. The combined diameter of delivery catheter plus mounted stent measures $1.57 \mathrm{~mm}$ in diameter. As the sleeve is withdrawn, the constrained device returns to its original unconstrained larger diameter and becomes anchored against the vessel wall $[1,2]$. Unconstrained stent diameter in this study was $2.5 \mathrm{~mm}$ in the $2 \mathrm{~mm}$ phantom and $3.0 \mathrm{~mm}$ in the $3 \mathrm{~mm}$ phantom.

From the Department of Cardiology, Thoraxcenter, Erasmus University Rotterdam, Rotterdam, The Netherlands.

Received July 31, 1991; revision accepted August 19, 1991.

Address requests to Dr. P.W. Serruys, Catheterization Laboratory, Thoraxcenter, Erasmus University Rotterdam, P.O. Box 1738, 3000 DR Rotterdam, The Netherlands.

Dr. Strauss is a Research Fellow of the Heart and Stroke Foundation of Canada.

Presented in part at the Sixty-Third American Heart Association Meeting, Dallas, Texas, November 12-15, 1990. 

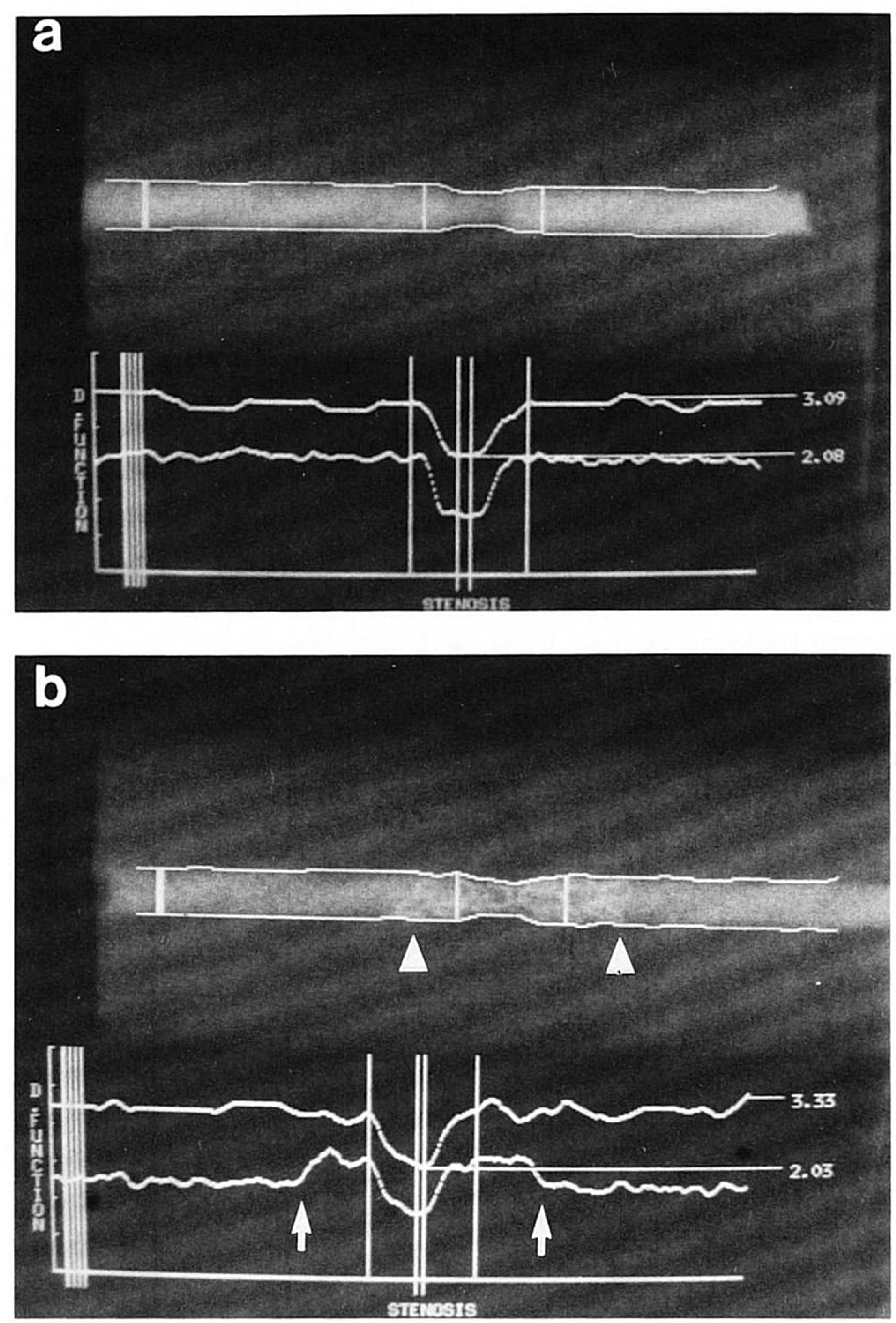

Fig. 1. Control (a) and Wiktor ${ }^{\mathbb{T} \mathrm{T}}$-containing (b) plexiglass phantoms $(3 \times 2 \mathrm{~mm})$ filled with $100 \%$ and $50 \%$ iopamidol contrast reagent, respectively. Graphs show the diameter function (upper curve) and the densitometric area function (lower curve). Outside vertical lines on the graph and rightward two vertical lines on the phantom are lesion boundaries. The inner two vertical lines represent the minimal points on the diameter and densitometric graphs, respectively. The multiple vertical lines in the left part of the graph and the leftward vertical line in the phantom represent the user-defined reference segment. The numbers in the graph represent the maximum and minimum diameters. The boundaries of the Wiktor ${ }^{i m}$ stent are visible in the phantom (arrowheads) and as a step-up in the densitometry graph (arrows). As a result of the Wiktor ${ }^{\text {itm }}$ stent contribution to the densitometry values, the minimal cross-sectional area determination is overestimated compared with the control phantom.

The Palmaz-Schatz ${ }^{\mathbb{T N}}$ coronary stent (Johnson and Johnson, Warren, NJ) is a balloon expandable slotted stainless steel tubular stent composed of two $7 \mathrm{~mm}$ segments of slotted tubes that are connected by a $1 \mathrm{~mm}$ bridging strut [4]. Each tube contains 12 rows of slots that are each $0.065 \mathrm{~mm}$ in diameter. The stent is mounted coaxially over commercially available balloon catheters. In this study the stent was cut at the bridging strut and only the tube was placed in the phantom.
The Wiktor ${ }^{\text {Til }}$ stent (Medtronic, Minneapolis, MN) is a balloon expandable tantalum helical coil stent that is constructed of a single tantalum wire $(0.127 \mathrm{~mm}$ in diameter) which is formed into a sinusoidal wave and wrapped into a helical coil structure. The prosthesis is crimped onto a deflated polyethylene angioplasty balloon and the maximal diameter of the balloon after inflation determines the ultimate size of the prosthesis after implantation [5].

\section{Angiographic Protocol}

Angiograms were obtained with the use of a Siemens Bicor X-ray system in the 5-in. cesium iodine image intensifier mode and a $0.8 \mathrm{~mm}$ focal spot of the X-ray tube. The focus-to-object distance was $90 \mathrm{~cm}$ and the object-to-image-intensifier distance was $13 \mathrm{~cm}$. In each study, the stent was introduced into the plexiglass phantom and positioned across the narrowing at the center of the phantom. All three stents completely covered the central narrowing and extended on either side to the reference segment. The phantoms were then filled with contrast medium, iopamidol-370, at a concentration of either $50 \%$ or $100 \%$.

Cinefilm was taken with an additional $17.5 \mathrm{~cm}$ thickness of plexiglass blocks $(12.5 \mathrm{~cm}$ anterior and $5 \mathrm{~cm}$ posterior to the models). The addition of the plexiglass blocks resulted in a more appropriate kilovoltage level $(82 \mathrm{kV})$ and in a scatter medium which more closely approximates the radiologic scatter in the human thorax during angiography. The studies were then repeated with the second concentration of the contrast reagent. The cinefilms were processed routinely and analyzed by the cardiovascular angiography analysis system (CAAS).

\section{Angiographic Analysis}

All cineangiograms were analyzed using the computer-assisted CAAS which has previously been discussed in detail [6-9]. The important steps will be briefly described. Any area of size of $6.9 \times 6.9 \mathrm{~mm}$ in a selected cineframe (overall dimensions $18 \times 24 \mathrm{~mm}$ ) encompassing the phantom or the desired arterial segment can be digitized by a high resolution CCD-camera with a resolution of $512 \times 512$ pixels and 8 bits of gray level. Contours of the "vessel" segments were determined automatically based on the weighted sum of the first and second derivative functions applied to the digitized brightness information along scanlines perpendicular to the local centerline directions of the perspex model. The centerline was manually defined by the user. A computer-derived estimation of the original dimension at the site of the narrowing is used to define the interpolated reference diameter. This technique is based on a computer-derived estimation of the original diameter values over the analyzed region (assuming there was no 
TABLE I. Videodensitometric Determination of Minimal Luminal Cross-Sectional Area in Plexiglass Phantoms Containing No (Control) or Three Different Metallic Stents*

\begin{tabular}{|c|c|c|c|c|c|c|c|c|}
\hline \multirow{2}{*}{$\begin{array}{l}\text { Iopamidol } \\
\text { concentration }\end{array}$} & \multirow{2}{*}{\multicolumn{2}{|c|}{$\begin{array}{c}\text { Reference } \\
\text { diameter } \\
(\mathrm{mm})\end{array}$}} & \multirow{2}{*}{$\begin{array}{c}\text { Stenosis } \\
\text { diameter } \\
(\mathrm{mm})\end{array}$} & \multicolumn{5}{|c|}{ Minimal luminal cross-sectional area $\left(\mathrm{mm}^{2}\right)$} \\
\hline & & & & True & Control & Palmaz-Schatz ${ }^{\top M}$ & Wallstent $^{\circledR}$ & Wiktor ${ }^{\mathrm{TM}}$ \\
\hline \multirow[t]{2}{*}{$100 \%$} & & 3 & 2 & 3.14 & $3.70 \pm .10$ & $3.55 \pm .10(-4 \%)$ & $3.93 \pm .11(+6 \%)$ & $4.17 \pm .23(+13 \%)$ \\
\hline & & 4 & 3 & 7.06 & $7.61 \pm .30$ & $8.19 \pm .25(+8 \%)$ & $7.78 \pm .50(+2 \%)$ & $8.33 \pm .21(+9 \%)$ \\
\hline \multirow[t]{2}{*}{$50 \%$} & & 3 & 2 & 3.14 & $3.14 \pm .13$ & $3.91 \pm .31(+25 \%)$ & $3.54 \pm .07(+13 \%)$ & $4.89 \pm .08(+56 \%)$ \\
\hline & & 4 & 3 & 7.06 & $7.65 \pm .21$ & $7.92 \pm .07(+4 \%)$ & $7.81 \pm .50(+2 \%)$ & $9.40 \pm .38(+23 \%)$ \\
\hline
\end{tabular}

$*(\%)=\%$ difference between the stented and control phantoms.

narrowing present) according to the diameter function. Calibration of the diameter data is performed by applying the automated contour detection technique to the known outer diameter of the plexiglass model.

The densitometric assessment, which relies on the relationship between pathlength of the X-rays through the segment and the resulting brightness values, requires a detailed analysis of the complete X-ray/cine/video chain, including the film development process.

For the first part of the chain, from the X-ray source to the output of the image intensifier, we use the LambertBeer law for the X-ray absorption and apply certain models for the X-ray source and the image intensifier. From the output of the image intensifier up to the brightness values in the digital image, a simple linear transfer function was used. Details of this technique have been described elsewhere [6-8]. The cross-sectional area of the perspex narrowing is then obtained as follows. Contours of the segment are detected by automated contour detection with the CAAS as previously described. From the measured diameters along the analyzed segment, the previously described diameter function is derived. On each scanline perpendicular to the local centerline direction of the vessel, a profile of brightness values is measured. This profile is transformed into an absorption profile by means of a simple logarithmic transfer function to correct for the Lambert-Beer law. The background contribution is estimated by computing the linear regression line through the background points directly left and right of the detected contours. Subtraction of this background portion from the absorption profile within the perspex contours yields the net cross-sectional absorption profile. Integration of this function gives a measure for the crosssectional area at the particular scanline. By repeating this procedure for all scanlines, the cross-sectional area function is obtained. An absolute reference densitometric area value can then be obtained by assuming a circular configuration in the reference segment and using the diameter measurements obtained from the diameter function. Figure 1 shows an example with the computed diameter function curve (upper curve) and area function curve (lower curve) displayed upon the video image. The minimal luminal cross-sectional area can then be calculated by the ratio of the absolute difference in brightness of the reference area and the narrowed segment, with the known cross-sectional area of the reference segment. The complete procedure has been evaluated with the cinefilms of perspex models of coronary obstructions that did not contain stents. In this protocol, one frame from each study was analyzed at least three times.

\section{RESULTS}

The densitometric assessment of the plexiglass models containing the stents is shown in Table I. The theoretical minimal luminal cross-sectional areas of a $2 \mathrm{~mm}$ and a 3 $\mathrm{mm}$ diameter phantom are 3.14 and $7.06 \mathrm{~mm}^{2}$, respectively. In the control phantom, the calculated minimal luminal cross-sectional area values ranged from $0-18 \%$ higher than the theoretical value in the $3 \times 2$ phantom, and was $8 \%$ higher in the $4 \times 3$ phantom (Fig. 1a). Only minor increases in minimal luminal cross-sectional area ( $\leq 8 \%$ were noted in the phantoms that contained the Wallstent ${ }^{\circledR}$ and Palmaz-Schatz ${ }^{(\mathbb{I I I}}$ stents compared with the control phantom except for the $3 \times 2$ phantom filled with $50 \%$ contrast medium (13 and $25 \%$ higher, respectively). The minimal luminal cross-sectional area in the phantom containing the Wiktor ${ }^{\text {(III) }}$ stent was moderately higher $(9-13 \%)$ than the control with $100 \%$ contrast medium. However, in the presence of $50 \%$ contrast medium, the Wiktor ${ }^{(\mathbb{N i})}$ stent had the most adverse effect (23-56\% higher than control values) in the determination of the minimal luminal cross-sectional area. This effect was most pronounced in the smaller $3 \times 2$ phantom (Fig. 1b).

\section{DISCUSSION}

This study illustrates two important points. First, in these particular models, densitometric determinations of minimal luminal cross-sectional area in control phantoms using the CAAS are overestimated. In most of the study conditions, this is a minor effect except for the smaller diameter phantom at $100 \%$ concentration. This degree of 

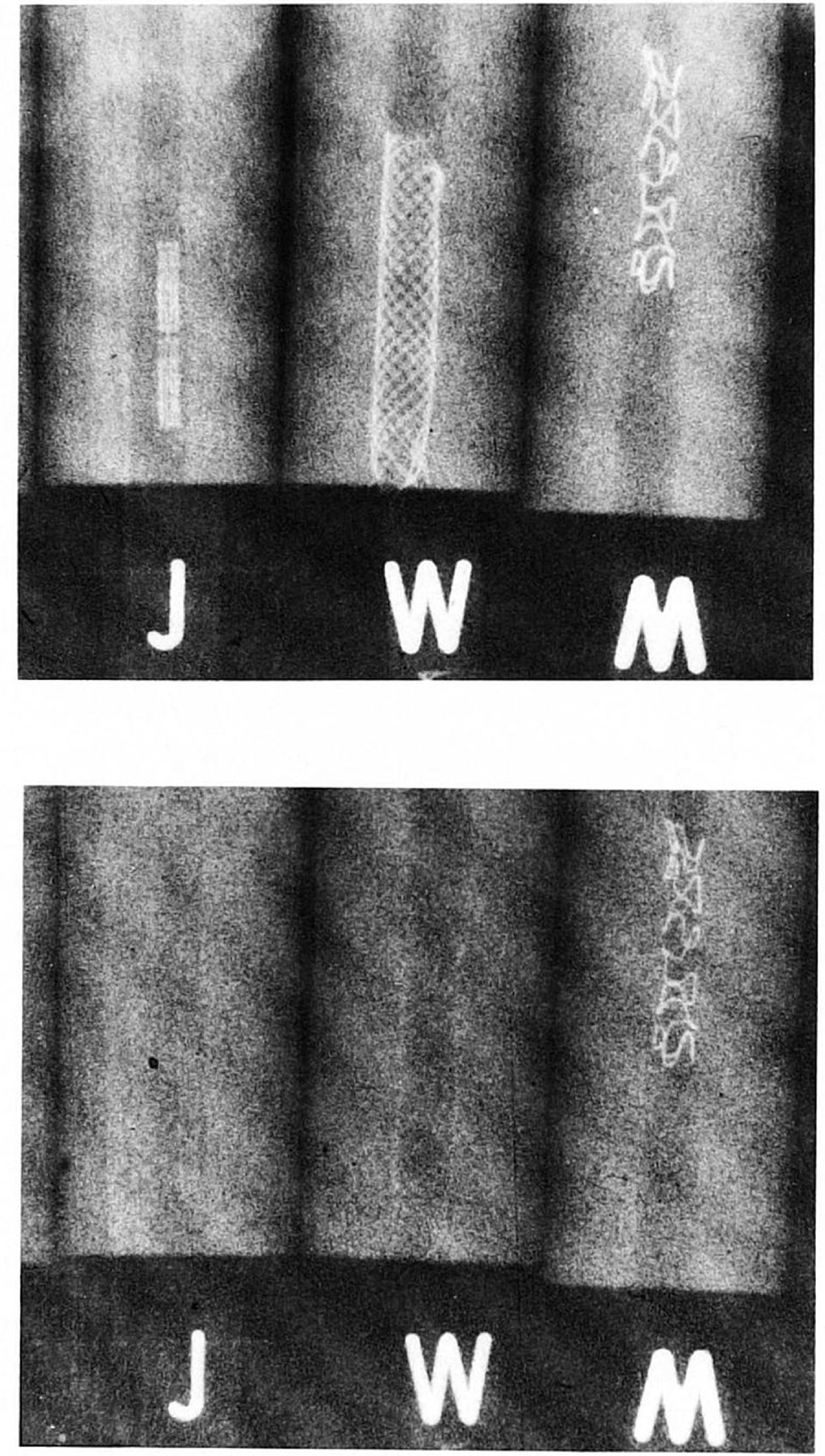

Fig. 2. Radiopacity of three stents at two kilovoltage levels in plexiglass phantoms without contrast. Top: $50 \mathrm{kV}$; bottom: 82 kV. $\mathbf{J}=$ Johnson and Johnson (Palmaz-Schatz $\left.{ }^{(m)}\right) ; W=$ Wallstent ${ }^{\circledR} ; M=$ Medtronic (Wiktor ${ }^{(\pi)}$ ). The Palmaz-Schatz ${ }^{(\mathbb{M}}$ stent was not expanded in the phantom.

error is in concordance with Whiting et al. [10]. Second, the addition of stents further contributes to an even spuriously higher measurement of the minimal luminal cross-sectional area, particularly with the Wiktor stent.

The radiographic assessment of coronary stents includes several important features. During the procedure, the ability to visualize the stent is important for proper stent deployment to ensure adequate coverage of a lesion. The Wiktor ${ }^{\text {TIN }}$ stent has a decisive advantage since tantalum is much more radiopaque than stainless steel, particularly at kilovoltage levels used in angiographic studies of the human thorax (Fig. 2). Densitometry has a theoretical advantage over edge detection since it is a more direct measurement of stenosis cross-sectional

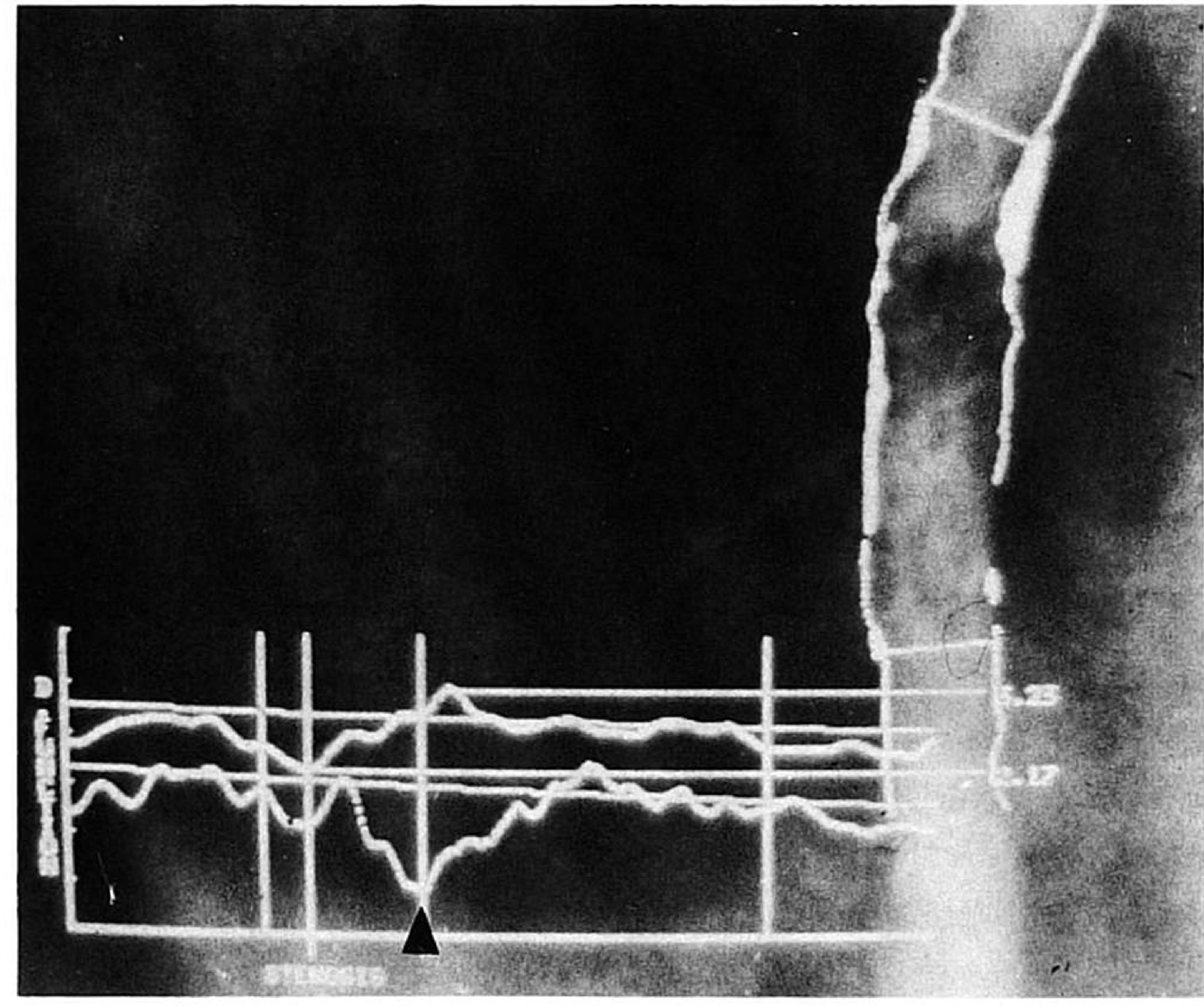

Fig. 3. Edge detection and densitometric analysis of an obstruction within a Wiktor ${ }^{(\pi)}$ stent in the right coronary artery. The four vertical lines in the graph were explained in Figure 1. The boundaries of the stent are represented by the two horizontal lines in the angiographic image and the two outer vertical lines in the graph. A discrepancy between the two graphs is present and most severe at the vertical line, which denotes the minimal densitometric value (arrowhead). The edge detection follows the outline of the stent and does not recognize the stenosis within the stent.

area. This parameter appears to be more closely related than vessel diameter to coronary flow of hemodynamics [11]. Thus densitometry may be an important method to evaluate the immediate angiographic result post stenting, and is particulary useful to analyze eccentric lesions since it is theoretically independent of the geometric shape. Although contour detection appears to be equally acceptable in Wallstent ${ }^{\circledR}$-implanted lesions [3], it has been unreliable in some cases of Wiktor implantations due to detection of the radiopaque stent wires as the border instead of the narrowing within the stent (Fig. 3).

This study suggests that densitometric assessment of Wiktor ${ }^{(M i}$-containing lesions is also affected by the radiographic properties of this particular stent, especially in situations where the contrast medium is diluted or the vessel is not well filled. This effect consistently resulted in an overestimation of the minimal luminal cross-sectional area by $9-56 \%$ from control values, depending upon the study conditions. Although the smallest differences between the Wiktor ${ }^{\mathbb{T M}}$-containing phantoms and control phantoms occurred with $100 \%$ contrast medium, these are also the conditions that resulted in the largest discrepancy between the control phantoms and the true values. Thus, the overall error in Wiktor ${ }^{(\mathbb{W})}$-containing phantoms with $100 \%$ contrast medium compared with 


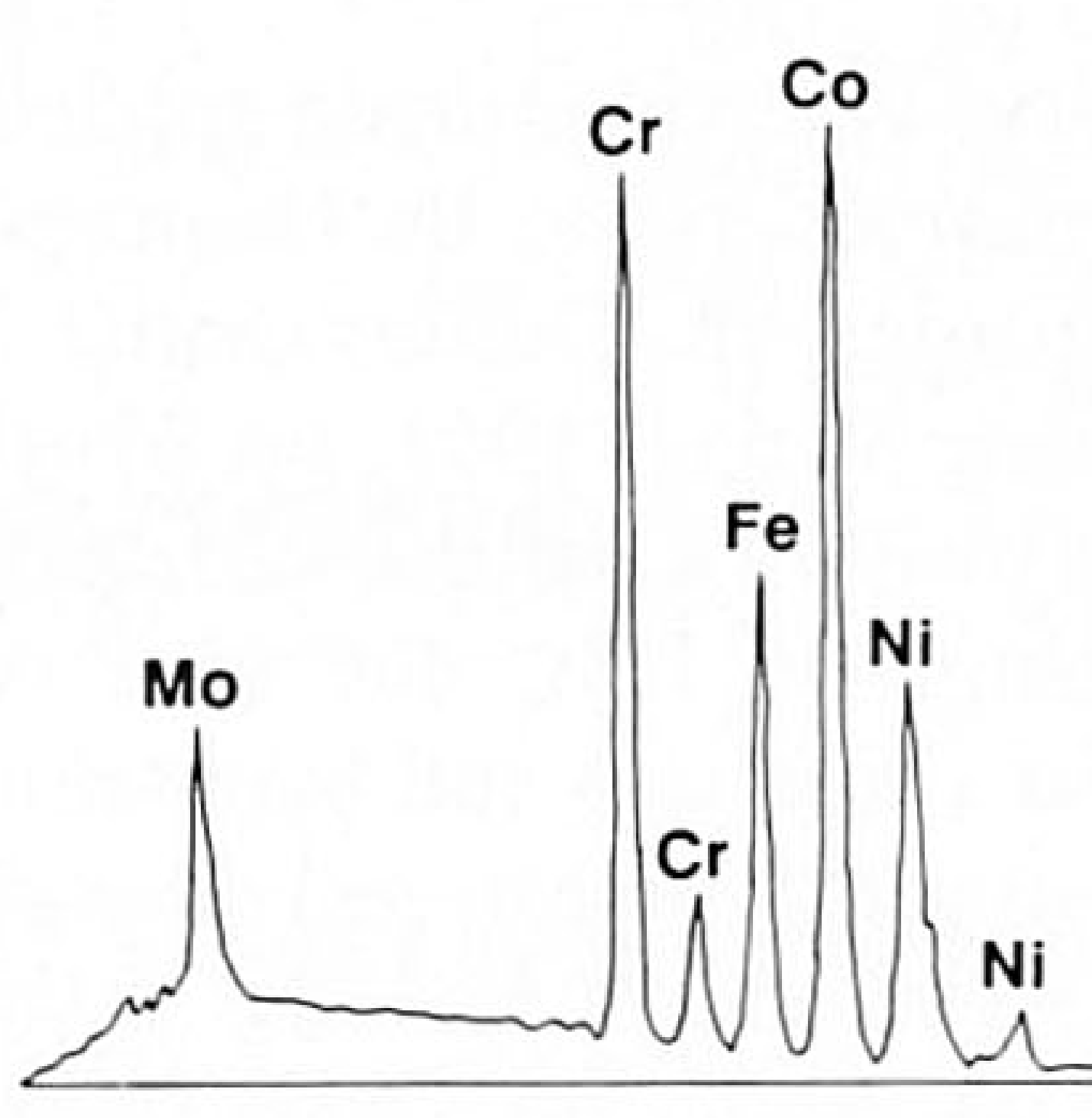

Wallstent (Medinvent)

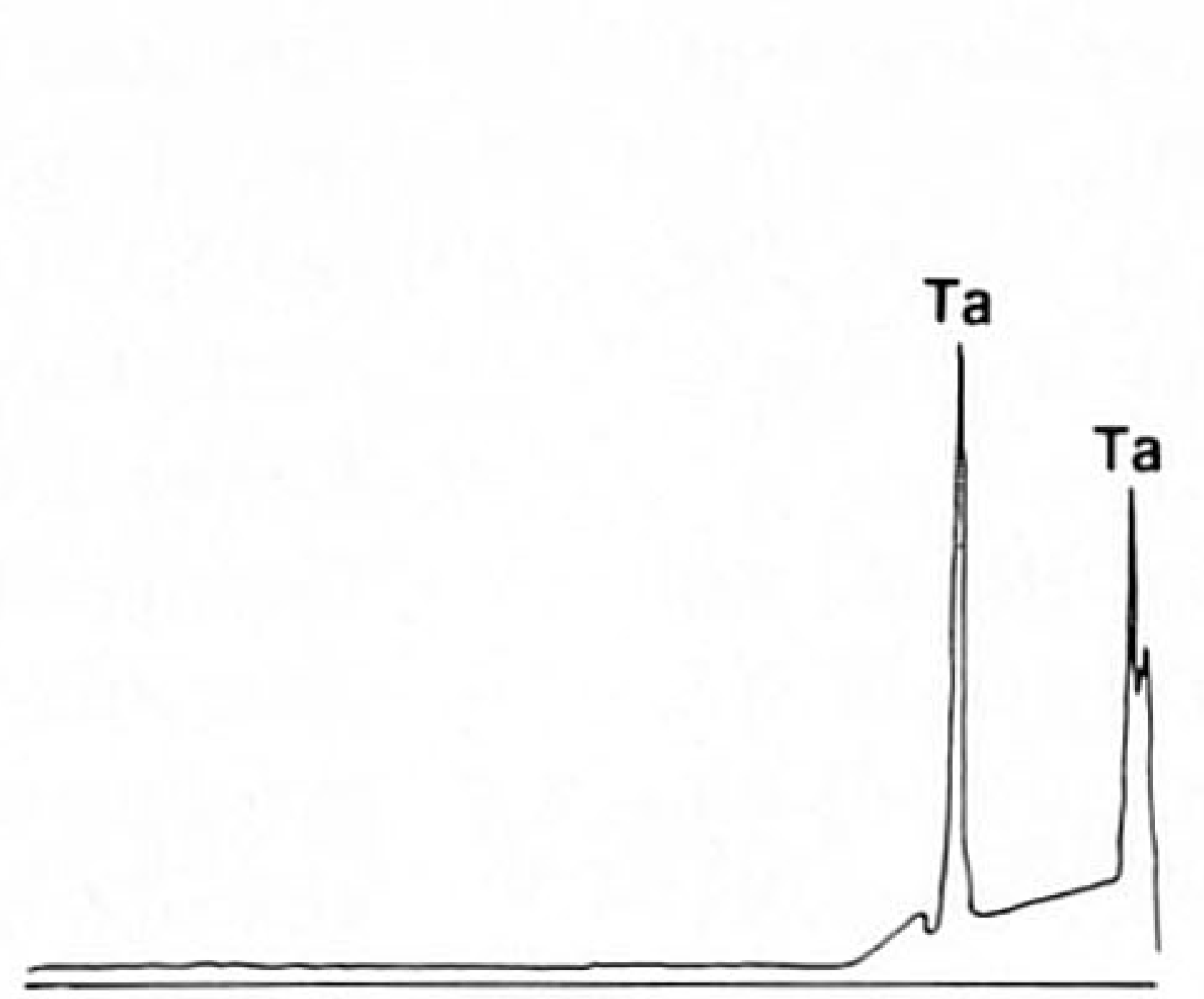

Wiktor stent (Medtronic)

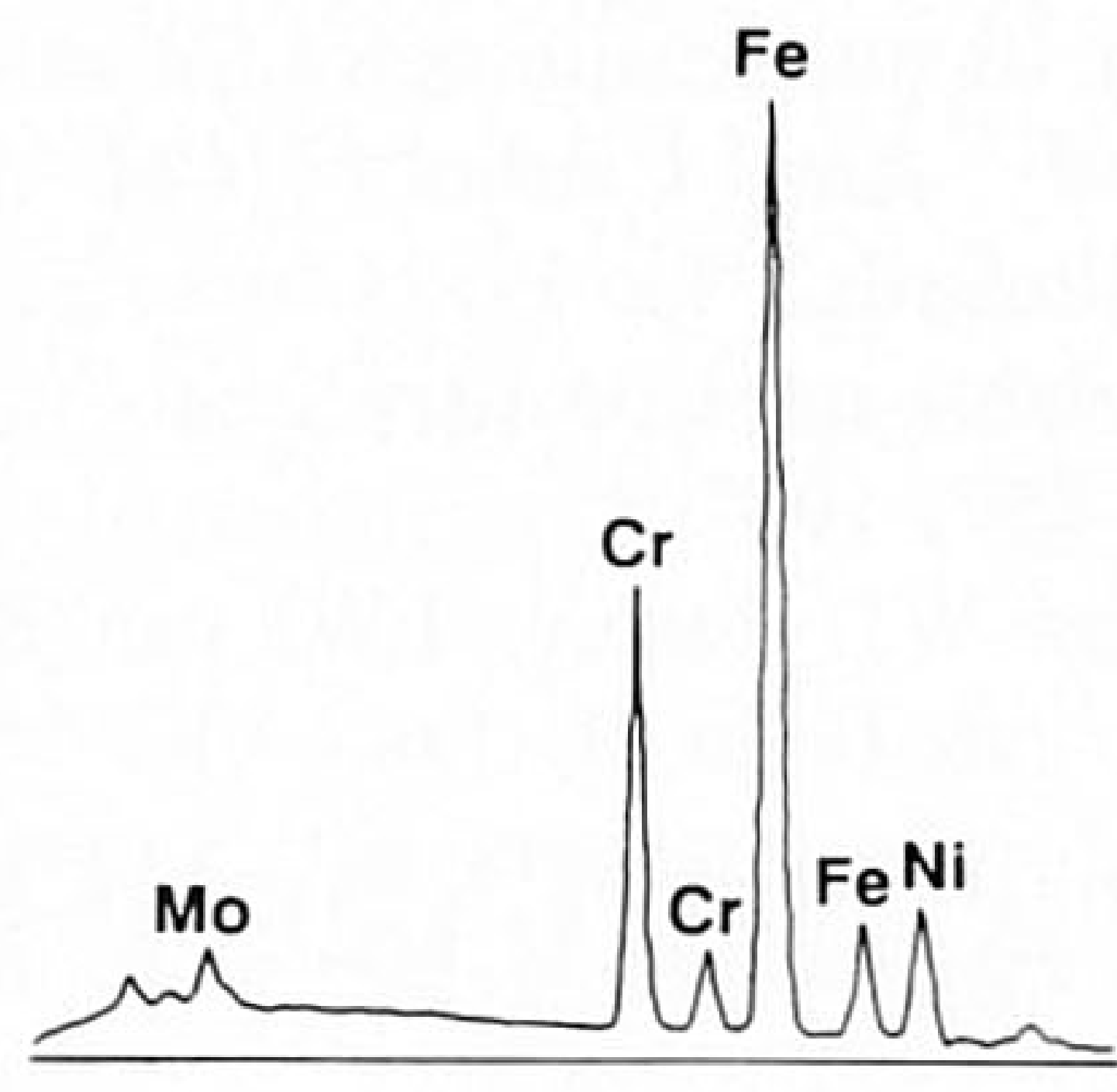

Palmaz-Schatz stent (J \& J)

Fig. 4. X-ray energy dispersion spectrometry identifies the compositon of the metals used in each of the three stents. $\mathrm{Mo}=$ molybdenum; $\mathrm{Cr}=$ cromium; $\mathrm{Co}=$ cobalt; $\mathrm{Fe}=$ iron, $\mathrm{Ni}=$ nickel; Ta = tantalum.

the true value was in the range of $18-33 \%$. At a lower contrast reagent concentration, the overestimation of minimal luminal cross-sectional area in the Wiktor (TiN stent was more pronounced compared with the control phantom, especially in the $2 \mathrm{~mm}$ phantom narrowing since there is relatively less contrast (and thus less iodine) present in the smaller phantom in comparison to the amount of tantalum contained in the stent wires. Therefore, the contribution of the stent wires to the overall densitometric value is more significant and results in the higher calculated values of the minimal luminal crosssectional area. This may have important consequences in follow-up cases of restenosis where the vessel lumen may be critically reduced in caliber.

The radiopacity of a stent is affected by several variables including the composition of the material (and its atomic number), the total surface areas of the wires in the stent, and the cross-sectional area of the stent wires encountered by the X-ray beam in any particular projection. The Wiktor ${ }^{\text {TII }}$ stent is made of radiopaque tantalum which, due to its higher atomic number than stainless steel, has a higher attenuation constant and thus increased radiopacity than the two stainless steelcontaining stents. X-ray energy dispersion spectrometry studies were done in all three stents to determine if other metals were present that could alter the attenuation coefficient (Fig. 4). The Wiktor ${ }^{(\mathrm{TM})}$ stent contained only tantalum. The compositions of the Wallstent ${ }^{\circledR}$ and the Palmaz-Schatz ${ }^{\text {(ii }}$ stent were very similar except for the presence of cobalt in the Wallstent ${ }^{\circledR}$. However, this would only minimally affect the attenuation constant.

The cross-sectional wire area of the stent is dependent on the number and thickness of the wire(s) at any particular point along the stent. This is more difficult to estimate in the Wiktor ${ }^{\text {(TiM }}$ stent due to the asymmetric and helical design of the stent and the orientation of the wire loops. Consequently, the cross-sectional area of the stent is not uniform at each segment over the length of the stent. It is estimated that at any one segment, a cross- section could contain from four to eight cut wires with a probable average of six wires. This corresponds to a cross-sectional area of the stent ranging from .0005 to $.001 \mathrm{~cm}^{2}$, and an average value of $.00075 \mathrm{~cm}^{2}$. The total wire cross-sectional areas of the Wallstent ${ }^{\circledR}$ and PalmazSchat ${ }^{(\mathbb{I}}{ }^{\mathrm{N}}$ stents, which are easier to calculate due to the more uniform design of each stent, are 0.00062 and $0.00039 \mathrm{~cm}^{2}$, respectively.

Since the composition and total wire cross-sectional area of the two stainless steel stents are nearly identical, it is not surprising that these two stents had a similar effect. This effect in general was quite consistent and minimal. Although the largest discrepancy between stainless steel stented and control phantoms occurred in the smaller phantom at $50 \%$ contrast concentration, these were the exact conditions that no overestimation of minimal luminal cross-sectional area occurred in the control phantoms compared with the true value.

In conclusion, densitometry may overestimate the minimal luminal cross-sectional area in stented vessels. Relatively minor and probably clinically insigificant changes occur in the Palmaz-Schatz ${ }^{\mathbb{( I N}}$ stent and the Wallstent ${ }^{\circledR}$. However, densitometric analysis of lesions containing the Wiktor ${ }^{(\text {IDI }}$ stent, particularly if the vessel is not well opacified, will result in serious overestimations of the minimal luminal cross-sectional area. Clinical trials that compare various stents or stenting to PTCA using densitometry should consider this effect in tantalum-containing stents.

\section{REFERENCES}

1. Sigwart U, Puel J, Mirkovitch V, Joffre F, Kappenberger L: Intravascular stents to prevent occlusion and restenosis after transluminal angioplasty. N Engl J Med 316:701-706, 1987.

2. Serruys PW, Strauss BH, Beatt KJ, Bertrand ME, Puel J, Rickards AF, Meier B, Kappenberger L, Goy JJ, Vogt P, Sigwart U: Angiographic follow-up after placement of a self-expanding coronary stent. N Engl J Med 324:13-17, 1991.

3. Strauss BH, Juilliere Y, Rensing BJ, Reiber JHC, Serruys PW: 
Edge detection versus densitometry for assessing coronary stenting quantitatively. Am J Cardiol 67:484-490, 1991.

4. Schatz RA, Palmaz JC, Tio FO, Garcia F, Garcia O, Reuter SR: Balloon-expandable intracoronary stents in the adult dog. Circulation 76:450-457, 1987.

5. van der Giessen WJ, Serruys PW, van Beusekom HMM, van Woerkens LJ, van Loon H, Soei LK, Strauss BH, Beatt KJ, Verdouw PD: Coronary stenting with a new, radiopaque, balloonexpandable endoprosthesis in pigs. Circulation 83:1788-1798, 1991.

6. Reiber JHC, Kooijman CJ, Slager CJ, Gerbrands JJ, Schuurbiers JHC, den Boer A, Wijns W, Serruys PW, Hugenholtz PG: Coronary artery dimensions from cineangiograms: Methodology and validation of a computer-assisted analysis procedure. IEEE Trans Med Imaging MI3:131-141, 1984.

7. Reiber JHC, Serruys PW, Slager CJ: " Quantitative Coronary and Left Ventricular Cineangiography. Methodology and Clinical Application.' Dordrecht: Martinus Nijhoff, 1986.

8. Reiber JHC, Slager CJ, Schuurbiers JCH, den Boer A, Gerbrands JJ, Troost GJ, Scholts B, Kooijman CJ, Serruys PW: Transfer functions of the X-ray cine video chain applied to digital processing of coronary cineangiograms. In: Heintzen PH, Brennecke R (eds): "Digital Imaging in Cardiovascular Radiology." New York: George Thieme Verlag, 1983, pp 80-104.

9. Reiber JHC, Serruys PW, Kooijman CJ, Wijns W, Slager CJ, Gerbrands JJ, Schuurbiers JCH, den Boer A, Hugenholtz PG: Assessment of short-, medium- and long-term variations in arterial dimensions from computer-assisted quantification of coronary cineangiograms. Circulation 71:280-288, 1985.

10. Whiting JS, Pfaff JM, Eigler NL: Advantages and limitation of videodensitometry in quantitative coronary angiography. In Reiber JHC, Serruys PW (eds): "Quantitative Coronary Arteriography.' Dordrecht: Kluwer Academic Publishers, 1991, pp 4354.

11. Harrison DG, White CW, Hiratzka LF, Doty DB, Barnes DH, Eastham CL, Marcus ML: The value of lesion cross-sectional area determined by quantitative coronary angiography in assessing the physiologic significance of proximal left anterior descending stenoses. Circulation 69:1111-1119, 1984. 